\title{
On Modules and Complexes without Self-extensions
}

\author{
Raymundo Bautista, Efrén Pérez
}

\begin{abstract}
Let $\Lambda$ be an artin algebra over a commutative artinian ring, $k$. If $M$ is a finitely generated left $\Lambda$-module, we denote by $\Omega(M)$ the kernel of $\eta_{M}: P_{M} \rightarrow M$ a minimal projective cover. We prove that if $M$ and $N$ are finitely generated left $\Lambda$-modules and $\operatorname{Ext}_{\Lambda}^{1}(M, M)=0, \operatorname{Ext}_{\Lambda}^{1}(N, N)=0$, then $M \cong N$ if and only if $M / \operatorname{rad} M \cong N / \operatorname{rad} N$ and $\Omega(M) \cong \Omega(N)$.

Now if $k$ is an algebraically closed field and $\left(d_{i}\right)_{i \in \mathbb{Z}}$ is a sequence of non negative integers almost all of them zero, then we prove that the family of objects $X \in \mathcal{D}^{b}(\Lambda)$, the bounded derived category of $\Lambda$, with $\operatorname{Hom}_{\mathcal{D}^{b}(\Lambda)}(X, X[1])=0$ and $\operatorname{dim}_{k} H^{i}(X)=d_{i}$ for all $i \in \mathbb{Z}$, has only a finite number of isomorphism classes (see [9]).
\end{abstract}

\section{Introduction}

Let $\Lambda$ be an artin algebra over a commutative artinian ring $k$. We denote by $\operatorname{Mod} \Lambda$ the category of left $\Lambda$-modules, by $\bmod \Lambda, \operatorname{proj} \Lambda$ we denote the full subcategories of Mod $\Lambda$ whose objects are respectively, the finitely generated $\Lambda$ modules and the finitely generated projective $\Lambda$-modules. By $\mathcal{D}^{b}(\Lambda)$ we denote the bounded derived category of $\Lambda$.

For $M \in \bmod \Lambda$, consider $P_{M}^{0} \stackrel{\eta_{M}}{\rightarrow} M$ a minimal projective cover and $\Omega(M)=$ $\operatorname{ker}\left(\eta_{M}\right)$. Here we prove the following: if $M, N$ are in $\bmod \Lambda$ and $\operatorname{Ext}_{\Lambda}^{1}(M, M) \cong$ $\operatorname{Ext}_{\Lambda}^{1}(N, N)=0$, then $M \cong N$ if and only if $M / \operatorname{rad} M \cong N / \operatorname{rad} N$ and $\Omega(M) \cong$ $\Omega(N)$.

For $M \in \bmod \Lambda$ with finite projective dimension consider a minimal projective resolution:

$$
0 \rightarrow P_{M}^{-m(M)} \rightarrow P_{M}^{-m(M)+1} \rightarrow \ldots \rightarrow P_{M}^{0} \stackrel{\eta_{M}}{\rightarrow} M \rightarrow 0 .
$$

Suppose that $M, N$ are in $\bmod \Lambda, \operatorname{Ext}_{\Lambda}^{1}(M, M) \cong \operatorname{Ext}_{\Lambda}^{1}(N, N)=0$ and for all $j>0, \operatorname{Ext}_{\Lambda}^{j}\left(M, \Omega^{j-1}(M)\right) \cong \operatorname{Ext}_{\Lambda}^{j}\left(N, \Omega^{j-1}(N)\right)=0$, then we prove using the result above that $M \cong N$ if and only if $m(M)=m(N)$ and for all $j, P_{M}^{-j} \cong P_{N}^{-j}$.

\footnotetext{
${ }^{1} 2000$ Mathematics subject classification: 16G60, 16G10, 16A46, 15A21.

Key words and phrases: Artin algebra, exact structure, extensions, lift category, projective resolution.
} 
Now let $\Lambda$ be a finite-dimensional algebra over an algebraically closed field $k$ and $\mathbf{d}=\left(d_{i}\right)_{i \in \mathbb{Z}}$ be a collection of non-negative integers with almost all $d_{i}=0$. Then the family $\mathcal{U}(\mathbf{d})$ of objects $X \in \mathcal{D}^{b}(\Lambda)$ such that $\operatorname{dim}_{k} H^{i}(X)=d_{i}$ for all $i \in \mathbb{Z}$ and $\operatorname{Hom}_{\mathcal{D}^{b}(\Lambda)}(X, X[1])=0$ has only a finite number of isomorphism classes in $\mathcal{D}^{b}(\Lambda)$. This result is closely related with Corollary 9 of [9].

For the proof of the above mentioned results we see that some problems involving upper bounded complexes of finitely generated projective $\Lambda$-modules with bounded homology can be reduced to problems involving complexes of fixed size of finitely generated projective $\Lambda$-modules (see Proposition 4.7). Then in this last case we can apply properties of lift categories introduced by W.W Crawley-Boevey in [6].

\section{Exact structures and exact subcategories}

Here we recall the notion of exact category. For $(\mathcal{A}, \mathcal{E})$, an exact category, $\mathcal{B}, \mathcal{C}$ full subcategories of $\mathcal{A}$ closed under extensions we consider the category of morphisms $f: W \rightarrow Z$ with $W$ an object of $\mathcal{B}$ and $W$ an object of $\mathcal{C}$. Then we introduce an exact structure on this category of morphisms.

Definition 2.1 Let $\mathcal{A}$ be an additive category. A pair of composable morphisms

$$
X \stackrel{i}{\rightarrow} Y \stackrel{d}{\rightarrow} Z
$$

is called exact if $i$ is kernel of $d$ and $d$ is cokernel of $i$.

Let $\mathcal{E}$ be a class of exact composable sequences $(i, d)$ in $\mathcal{A}$ closed under isomorphisms; we call $(i, d) \in \mathcal{E}$ a conflation, $i$ an inflation and $d$ a deflation.

$\mathcal{E}$ is an exact structure if it satisfies the following axioms:

$K 1) 1_{0}$ is a deflation.

$K 2)$ Composition of deflations is a deflation.

$K 3)$ For every $h \in \mathcal{A}\left(Z, Z_{0}\right)$ and all deflation $d_{0} \in \mathcal{A}\left(Y_{0}, Z_{0}\right)$ there exists a pullback

$$
\begin{array}{ccc} 
& d & \\
Y & \rightarrow & Z \\
g \downarrow & d_{0} & \downarrow^{h} \\
Y_{0} & \rightarrow & Z_{0}
\end{array}
$$

where $d$ is a deflation

$\left.K 3^{o p}\right)$ For every $f \in \mathcal{A}\left(X, X_{0}\right)$ and all inflation $i \in \mathcal{A}(Y, Z)$ there exists a pushout

$$
\begin{array}{ccc}
X & i & \\
f \downarrow & Y \\
X_{0} & i_{0} & \downarrow^{g} \\
& \rightarrow & Y_{0}
\end{array}
$$

where $i_{0}$ is an inflation 
K4) Retractions in $\mathcal{A}$ have kernels.

In this situation we say that $(\mathcal{A}, \mathcal{E})$ is an exact category. For simplicity, if $\mathcal{E}$ is an exact structure we use extension instead of conflation.

Remark 2.2 It is known ([7]) that the above axioms imply their duals.

Moreover, in an exact category the next claims are true ([7]); if $d d^{\prime}$ is a deflation then $d$ is a deflation, if $i^{\prime} i$ is an inflation then $i$ is an inflation.

Also, $K 3$ induces a diagram of extensions

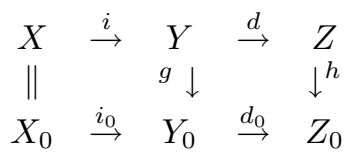

where the right square is a pullback and a pushout, and $K 3^{o p}$ a diagram of extensions

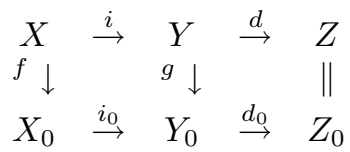

where the left square is a pullback and a pushout.

The following is a well known result.

Proposition 2.3 Let $(\mathcal{A}, \mathcal{E})$ be an exact category, and $\mathcal{B}$ a full subcategory closed under direct summands and extensions. Then $\left(\mathcal{B}, \mathcal{E}_{\mathcal{B}}\right)$ is an exact structure, where $\mathcal{E}_{\mathcal{B}}$ is the restriction of the class $\mathcal{E}$ to $\mathcal{B}$.

Definition 2.4 Let $\mathcal{A}$ be a category, and $\mathcal{B}$ and $\mathcal{C}$ subcategories of $\mathcal{A}$. We define the category $\operatorname{Morph}(\mathcal{B}, \mathcal{C})$ as follows: the objects are the morphisms $f: X \rightarrow Y$ in $\mathcal{A}$ such that $X \in \mathcal{B}$ and $Y \in \mathcal{C}$, and a morphism from $f: X \rightarrow Y$ to $f^{\prime}: X^{\prime} \rightarrow Y^{\prime}$ is a pair $(u, v)$ of morphisms $u: X \rightarrow X^{\prime}$ in $\mathcal{B}$ and $v: Y \rightarrow Y^{\prime}$ in $\mathcal{C}$ such that $f^{\prime} u=v f$.

Proposition 2.5 Let $(\mathcal{A}, \mathcal{E})$ be an exact category, and $\mathcal{B}$ and $\mathcal{C}$ full subcategories of $\mathcal{A}$ closed under direct summands and extensions. Then $\left(\operatorname{Morph}(\mathcal{B}, \mathcal{C}), \mathcal{E}_{\mathcal{C}}^{\mathcal{B}}\right)$ is an exact category, where $\mathcal{E}_{\mathcal{C}}^{\mathcal{B}}$ is the class of the pairs $\left(\left(u_{0}, v_{0}\right),\left(u_{1}, v_{1}\right)\right)$ such that $\left(u_{0}, u_{1}\right) \in \mathcal{E}_{\mathcal{B}}$ and $\left(v_{0}, v_{1}\right) \in \mathcal{E}_{\mathcal{C}}$.

Proof. Let $f_{1}: X_{1} \rightarrow Y_{1}, f_{2}: X_{2} \rightarrow Y_{2}$ and $f_{3}: X_{3} \rightarrow Y_{3}$ be objects in $\operatorname{Morph}(\mathcal{B}, \mathcal{C})$ and $\eta: f_{1} \stackrel{\left(u_{1}, v_{1}\right)}{\longrightarrow} f_{2} \stackrel{\left(u_{2}, v_{2}\right)}{\longrightarrow} f_{3}$ an element of $\mathcal{E}_{\mathcal{C}}$.

Clearly $\mathcal{E}_{\mathcal{C}}^{\mathcal{B}}$ is closed under isomorphisms.

Now we check that $\eta$ is an exact pair.

Suppose we have an object $f: X \rightarrow Y$ and a morphism $(u, v): f \rightarrow f_{2}$ in $\operatorname{Morph}(\mathcal{B}, \mathcal{C})$ such that $\left(u_{2}, v_{2}\right)(u, v)=0$. Then there exist unique morphisms $s: X \rightarrow X_{1}$ and $t: Y \rightarrow Y_{1}$ in $\mathcal{A}$ such that $u_{1} s=u$ and $v_{1} t=v$. We have $v_{1}\left(f_{1} s-t f\right)=f_{2} u_{1} s-v f=0$, then $f_{1} s=t f$ following that $(s, t)$ is a morphism.

The proof of $\left(u_{2}, v_{2}\right)$ being cokernel is dual. 
$K 1, K 2$ and $K 4$ are immediate from the proposition 2.3 .

In order to prove $K 3$, let $f$ be as before, take $(u, v): f \rightarrow f_{3}$ a morphism in $\operatorname{Morph}(\mathcal{B}, \mathcal{C})$, and consider the pullback diagrams

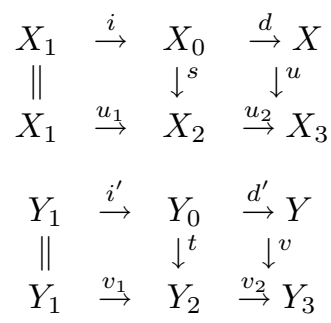

for the property of the pullback, there exists a unique morphism $f_{0}: X_{0} \rightarrow Y_{0}$ such that $d^{\prime} f_{0}=f d$ and $t f_{0}=f_{2} s$.

Suppose there is an object $g: W \rightarrow Z$ and morphisms $\left(\lambda_{1}, \lambda_{2}\right): g \rightarrow f_{2}$ and $\left(\mu_{1}, \mu_{2}\right): g \rightarrow f_{3}$ in $\operatorname{Morph}(\mathcal{B}, \mathcal{C})$ such that $\left(u_{2}, v_{2}\right)\left(\lambda_{1}, \lambda_{2}\right)=\left(\mu_{1}, \mu_{2}\right)(u, v)$. Then, by the pullback property, there exist unique morphisms $\alpha: W \rightarrow X_{0}$ and $\beta: Z \rightarrow Y_{0}$ in $\mathcal{A}$ such that $s \alpha=\lambda_{1}, d \alpha=\mu_{1}, t \beta=\lambda_{2}$ and $d^{\prime} \beta=\mu_{2}$.

Now observe that $d^{\prime}\left(f_{0} \alpha-\beta g\right)=f d \alpha-\mu_{2} g=0$ and $t\left(f_{0} \alpha-\beta g\right)=$ $f_{2} s \alpha-\lambda_{2} g=f_{2} \lambda_{1}-\lambda_{2} g=0$. By the pullback property $(\alpha, \beta)$ is a morphism in $\operatorname{Morph}(\mathcal{B}, \mathcal{C})$.

A dual argument proves $K 3^{o p}$.

\section{$3 \quad$ Lift categories}

Here we recall the properties of lift categories needed in our work. Now suppose $\mathcal{A}$ is a Krull-Schmidt category and $X, Y$ are objects in $\mathcal{A}$. We consider the category of morphisms in $\mathcal{A}, f: W \rightarrow Z$, with $W$ a finite direct sum of direct summands of $X$ and $Z$ a finite direct sum of direct summands of $Y$. We see that this category of morphisms can be seen as a lift category.

Definition 3.1 A lift pair $(R, \xi)$ is given by a ring $R$ and an exact sequence of $R$-bimodules

$$
\xi: 0 \quad \rightarrow \quad M \stackrel{i}{\rightarrow} E \stackrel{\pi}{\rightarrow} R \rightarrow 0
$$

Definition 3.2 Given a lift pair $(R, \xi)$ we define the lift category $\xi(R)$ as follows: the objects are pairs $(P, e)$ where $P$ is a projective $R$-module and $e: P \rightarrow E \otimes_{R} P$ is an $R$-morphism such that the composition

$$
P \quad \stackrel{e}{\rightarrow} \quad E \otimes_{R} P \stackrel{\pi \otimes 1}{\rightarrow} \quad R \otimes_{R} P \stackrel{\cong}{\rightrightarrows} P
$$

is $1_{P}$. A morphism $f:(P, e) \rightarrow\left(P^{\prime}, e^{\prime}\right)$ is an $R$-morphism $f: P \rightarrow P^{\prime}$ such that the following diagram is commutative 


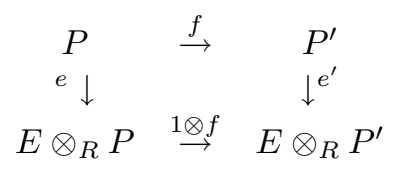

An object $(P, e)$ in $\xi(R)$ is called finite if and only if $P$ is a finitely generated $R$-module.

Definition 3.3 Let $\xi(R)$ be a lift category and $F_{0}: \xi(R) \rightarrow R$-Proj the forgetful functor. We define $\mathcal{H}$ as the class of sequences $Y \stackrel{i}{\rightarrow} Z \stackrel{d}{\rightarrow} X$ in $\xi(R)$ such that the sequence $0 \rightarrow F_{0}(Y) \rightarrow F_{0}(Z) \rightarrow F_{0}(X) \rightarrow 0$ is exact. It is known ([5]) that $\mathcal{H}$ is an exact structure, and we will always associate this structure to any lift category.

Definition 3.4 Let $\mathcal{A}$ be an additive category. For $X$ an object in the category $\mathcal{A}$ and $\Gamma_{X}=\operatorname{End}_{\mathcal{A}}(X)^{o p}$, we denote by $G_{X}: \mathcal{A} \rightarrow \operatorname{Mod} \Gamma_{X}$ the evaluation functor $\operatorname{Hom}_{\mathcal{A}}(X, ?)$.

Proposition 3.5 (II.2.1 [1]) Let $\mathcal{A}$ be an additive Krull-Schmidt category with splitting idempotents. Let $X$ be in $\mathcal{A}$, then:

1. $G_{X}: \operatorname{Hom}_{\mathcal{A}}(W, Z) \rightarrow \operatorname{Hom}_{\Gamma_{X}}\left(G_{X}(W), G_{X}(Z)\right)$ is an isomorphism for $W$ in add $X$ and $Z$ in $\mathcal{A}$.

2. If $W$ is in addX then $G_{X}(W)$ is in $\mathcal{P}\left(\Gamma_{X}\right)$.

3. $\left.G_{X}\right|_{\operatorname{add} X}: a d d X \rightarrow \mathcal{P}\left(\Gamma_{X}\right)$ is an equivalence of categories.

Remark 3.6 Let $\mathcal{A}$ be an additive Krull-Schmidt category with splitting idempotents and $X, Y \in \mathcal{A}$.

Assume $X=\oplus_{i}^{n} X_{i}$ and $Y=\oplus_{t}^{m} Y_{t}$, where each summand is indecomposable and the summands are pairwise non-isomorphic. It is clear that $G_{X}(Y)$ is a $\Gamma_{X}-\Gamma_{Y}$-bimodule.

Let $e_{i}$ be the idempotent of $\Gamma_{X}$ determined by $X_{i}$ and $W \cong \oplus_{i}^{n} c_{i} X_{i}$, then $\amalg_{i}^{n} c_{i} \Gamma_{X} e_{i} \cong G_{X}(W)$ as $\Gamma_{X}-$ modules.

Now let $Z$ be in add $Y$, there is a $\Gamma_{X}$-isomorphism $\phi_{Z}: G_{X}(Y) \otimes_{\Gamma_{Y}}$ $G_{Y}(Z) \rightarrow G_{X}(Z)$ given by $u \otimes v \mapsto v u$.

Moreover, if $g: Z \rightarrow Z^{\prime}$ is a morphism in $a d d Y$ we have a commutative diagram of $\Gamma_{X}$-modules:

$$
\begin{aligned}
& \begin{array}{ccc}
G_{X}(Y) \otimes_{\Gamma_{Y}} G_{Y}(Z) & \stackrel{\phi_{Z}}{\rightarrow} & \begin{array}{c}
G_{X}(Z) \\
\downarrow^{1 \otimes G_{Y}(g)}
\end{array} \\
& \downarrow^{G_{X}(g)}
\end{array} \\
& G_{X}(Y) \otimes_{\Gamma_{Y}} G_{Y}\left(Z^{\prime}\right) \stackrel{\phi_{Z^{\prime}}}{\longrightarrow} G_{X}\left(Z^{\prime}\right)
\end{aligned}
$$

This remark ends with the next convention: if $\mathcal{A}$ is an additive Krull-Schmidt category with splitting idempotents, it always has the exact structure of the trivial extensions; if it is not indicated in other way, we think in $\mathcal{A}$ as an exact category with trivial extensions. 
Proposition 3.7 Let $\mathcal{A}$ be an additive Krull-Schmidt category with splitting idempotents, $X$ and $Y$ in $\mathcal{A}$ where $X=\oplus_{i}^{n} X_{i}, Y=\oplus_{t}^{m} Y_{t}$, and $\left(X_{1}, \ldots, X_{n}\right)$ and $\left(Y_{1}, \ldots, Y_{m}\right)$ are pairwise non-isomorphic indecomposable objects in $\mathcal{A}$. Then there is an equivalence of categories $\Theta:$ Morph $(\operatorname{add} X$, add $Y) \rightarrow \xi_{Y}^{X}\left(R_{Y}^{X}\right)$, where the lift category is determined by the splitting lift pair $\left(R_{Y}^{X}, \xi_{Y}^{X}\right)$ :

$0 \rightarrow\left(\begin{array}{cc}0 & \operatorname{Hom}_{\mathcal{A}}(X, Y) \\ 0 & 0\end{array}\right) \rightarrow\left(\begin{array}{cc}\Gamma_{X} & \operatorname{Hom}_{\mathcal{A}}(X, Y) \\ 0 & \Gamma_{Y}\end{array}\right) \rightarrow\left(\begin{array}{cc}\Gamma_{X} & 0 \\ 0 & \Gamma_{Y}\end{array}\right) \rightarrow 0$

Moreover the functor $\Theta$ is an exact functor, i.e., it sends $\mathcal{E}_{\text {add } Y}^{a d d}$-extensions to $\mathcal{H}$-extensions in $\xi_{Y}^{X}\left(R_{Y}^{X}\right)$.

Proof. $\xi_{Y}^{X}\left(R_{Y}^{X}\right)$ is equivalent to the category of $\Gamma_{X}$-morphisms $t: P_{X} \rightarrow$ $\operatorname{Hom}_{\mathcal{A}}(X, Y) \otimes_{\Gamma_{Y}} P_{Y}$, where $P_{X}$ and $P_{Y}$ are $\Gamma_{X}$-projective and $\Gamma_{Y}$-projective modules respectively.

For $\alpha \in \operatorname{Hom}_{\mathcal{A}}(W, Z)$ in Morph $(a d d X, a d d Y)$ put $\Theta(\alpha)=\phi_{Z}^{-1} G_{X}(\alpha)$. Let $\alpha^{\prime} \in \operatorname{Hom}_{\mathcal{A}}\left(W^{\prime}, Z^{\prime}\right)$ and $(f, g): \alpha \rightarrow \alpha^{\prime}$ be in Morph (addX, add $\left.Y\right)$ we define $\Theta(f, g)=\left(G_{X}(f), 1_{\operatorname{Hom}_{\mathcal{A}}(X, Y)} \otimes G_{Y}(g)\right)$.

The functor $\Theta$ is dense by proposition 3.5.3. By proposition 3.5.1 and remark 3.6 it follows that $\Theta$ is a full and faithful functor. The exactness is immediate.

\section{Complexes and projective resolutions}

In this section we see some relations between the homotopy category of upper bounded complexes over $\operatorname{Proj} \Lambda$ with bounded homology and the complexes of fixed size over Proj $\Lambda$ (see proposition 4.7).

Notation 4.1 Let $\mathcal{A}$ be an additive category.

1. Denote by $C(\mathcal{A})$ the category of complexes over $\mathcal{A}$, a complex $X \in C(\mathcal{A})$ is a sequence $\left(X^{i}, d_{X}^{i}\right)_{i \in \mathbb{Z}}$ with $X^{i} \in \mathcal{A}$ and $d_{X}^{i}: X^{i} \rightarrow X^{i+1}$ morphisms in $\mathcal{A}$ such that $d_{X}^{i+1} d_{X}^{i}=0$. If $X=\left(X^{i}, d_{X}^{i}\right)_{i \in \mathbb{Z}}$ and $Y=\left(Y^{i}, d_{Y}^{i}\right)_{i \in \mathbb{Z}}$ are two complexes, a morphism $f: X \rightarrow Y$ is a sequence of morphisms in $\mathcal{A}$, $\left(f^{i}: X^{i} \rightarrow Y^{i}\right)_{i \in \mathbb{Z}}$ such that $f^{i+1} d_{X}^{i}=d_{Y}^{i} f^{i}$.

2. If $X \in C(\mathcal{A})$ and $s \in \mathbb{Z}$, the translation functors are defined by $(X[s])^{i}=$ $X^{i+s}$ and $\left(d_{X[s]}\right)^{i}=(-1)^{s}\left(d_{X}\right)^{i+s}$.

3. Recall that $f, g \in \operatorname{Hom}_{C(\mathcal{A})}(X, Y)$ are homotopic if there are morphisms $h^{i}: X^{i} \rightarrow Y^{i-1}$ such that $f^{i}-g^{i}=h^{i+1}\left(d_{X}\right)^{i}+\left(d_{Y}\right)^{i-1} h_{i}$ for all $i \in \mathbb{Z}$. For $X$ and $Y$ complexes in $C(\mathcal{A})$, we denote by $\operatorname{Hom}_{K}(X, Y)$ the homomorphisms in the homotopy category.

\section{Definition 4.2}


1. We denote by $C^{\leq m}(\mathcal{A})$ the full subcategory of complexes $X \in C(\mathcal{A})$ such that $X^{i}=0$ for $i>m$, and by $C^{\geq m}(\mathcal{A})$ the full subcategory of complexes $X \in C(\mathcal{A})$ such that $X^{i}=0$ for $i<m$. For $C^{[m, n]}(\mathcal{A})$ we mean the intersection $C^{\leq n}(\mathcal{A}) \cap C^{\geq m}(\mathcal{A})$.

2. Let us denote by $t_{m}: C(\mathcal{A}) \rightarrow C^{\leq m}(\mathcal{A})$ the "erase at right" functor, given in objects as $t_{m}(X)=\left(\left(t_{m}(X)\right)^{i}, d_{t_{m}(X)}^{i}\right)$ :

$$
\left(t_{m}(X)\right)^{i}=\left\{\begin{array}{cl}
X^{i} & \text { if } m \geq i \\
0 & \text { otherwise }
\end{array}\right\}, \quad\left(d_{t(m)(X)}\right)^{i}=\left\{\begin{array}{cl}
d_{X}^{i} & \text { if } i<m \\
0 & \text { otherwise }
\end{array}\right\}
$$

If $f: X \rightarrow Y$ is a morphism of complexes then $t_{m}(f)=\left(\left(t_{m}(f)^{i}\right)\right.$ where

$$
t_{m}(f)^{i}=\left\{\begin{array}{cc}
f^{i} & \text { if } m \geq i \\
0 & \text { otherwise }
\end{array}\right\}
$$

Dually we define the functor $l_{m}: C(\mathcal{A}) \rightarrow C^{\geq m}(\mathcal{A})$ "erase at left".

Now we denote by $s_{m}: C(\mathcal{A}) \rightarrow C^{\geq m}(\mathcal{A})$ the functor "erase and pull", given in objects as follows:

$$
s_{m}(X)^{i}=\left\{\begin{array}{cl}
X^{i+1} & \text { if } i \geq m \\
0 & \text { otherwise }
\end{array}\right\}, \quad d_{s_{m}(X)}^{i}=\left\{\begin{array}{cl}
d_{X}^{i+1} & \text { if } i \geq m \\
0 & \text { otherwise }
\end{array}\right\}
$$

If $f: X \rightarrow Y$ is a morphism of complexes then:

$$
\left(s_{m}(f)\right)^{i}=\left\{\begin{array}{cc}
f^{i+1} & \text { if } i \geq m \\
0 & \text { otherwise }
\end{array}\right\}
$$

3. We define the m-bending functor

$$
\urcorner_{m}: C(\mathcal{A}) \rightarrow \operatorname{Morph}\left(C^{\leq m}(\mathcal{A}), C^{\geq m}(\mathcal{A})\right)
$$

as follows: $\urcorner_{m}(X)=\left(u^{i}\right): t_{m}(X) \rightarrow s_{m}(X)$ where $u^{m}=d_{X}^{m}$ and $u^{i}=0$ for $i \neq m$, and for a morphism $f: X \rightarrow Y$ we have $\rceil_{m}(f)=$ $\left(t_{m}(f), s_{m}(f)\right)$.

Remark 4.3 In $C(\mathcal{A})$ there is a natural exact structure $\mathcal{E}$ given by composable pairs $f: X \rightarrow Y, g: Y \rightarrow Z$ such that $0 \rightarrow X^{i} \stackrel{f^{i}}{\rightarrow} Y^{i} \stackrel{g^{i}}{\rightarrow} Z^{i} \rightarrow 0$ are split exact for all $i \in \mathbb{Z}$. The exact category $(C(\mathcal{A}), \mathcal{E})$ has enough projectives and enough injectives, moreover the projectives coincide with the injectives. The stable category $\underline{C}(\mathcal{A})$, which is the category with the same objects as $C(\mathcal{A})$ and morphisms those in $C(\mathcal{A})$ modulo the morphisms which are factorized through projectives, coincides with $K(\mathcal{A})$, the homotopy category of $C(\mathcal{A})$. 
Remark 4.4 Observe that by proposition 2.3 , there are canonical induced exact structures on $C^{\leq n}(\mathcal{A}), C^{\geq m}(\mathcal{A})$ and on $C^{\leq n}(\mathcal{A}) \cap C^{\geq m}(\mathcal{A})$.

The definitions introduced in this section allow us to describe in a short way the next result.

Lemma 4.5 $\urcorner_{m}: C(\mathcal{A}) \rightarrow$ Morph $\left(C^{\leq m}(\mathcal{A}), C^{\geq m}(\mathcal{A})\right)$ is an exact isomorphism of categories for any $m \in \mathbb{Z}$.

Since $(C(\operatorname{Proj} \Lambda), \mathcal{E})$ is an exact category for $X, Y$ in $C(\operatorname{Proj} \Lambda)$ and $n$ a positive integer we have the extension group $\operatorname{Ext}_{C(\operatorname{Proj} \Lambda)}^{n}(X, Y)$, see 12.3 of [8]. For $n=1, \operatorname{Ext}_{C(\operatorname{Proj} \Lambda)}^{1}(X, Y)$ coincides, as in abelian categories, with the set of equivalence classes of sequences in $\mathcal{E}, Y \rightarrow E \rightarrow X$. For $X, Y$ in $\mathcal{U}$, a full subcategory of $C(\operatorname{Proj} \Lambda)$ closed under extensions, we have the extension groups $\operatorname{Ext}_{\mathcal{U}}^{j}(X, Y)$ corresponding to the induced exact structure on $\mathcal{U}$. Through the paper we say that $W$ an object of $\mathcal{U}$, is projective (respectively injective ) if for all $X \in \mathcal{U}, \operatorname{Ext}_{\mathcal{U}}^{1}(W, X)=0$ (respectively $\left.\operatorname{Ext}_{\mathcal{U}}^{1}(X, W)=0\right)$.

For $Y$ in the category $C(\operatorname{Proj} \Lambda)$ there is an exact sequence in $\mathcal{E}, Y \rightarrow W \rightarrow$ $Y[1]$ with $W$ injective. Then for all $X \in C(\operatorname{Proj} \Lambda)$ we have $\operatorname{Ext}_{C(\operatorname{Proj} \Lambda)}^{1}(X, Y) \cong$ $\operatorname{Hom}_{C(\operatorname{Proj} \Lambda)}(X, Y[1]) / I(X, Y[1]) \cong \operatorname{Hom}_{K}(X, Y[1])$, where $I(X, Y[1])$ is the subspace of morphisms which are factorized through injectives. If $u$ is any integer and $P$ a projective $\Lambda$-module we define the complex $J_{u}(P)$ in $C(\operatorname{Proj} \Lambda)$ as follows: $J_{u}(P)^{i}=0$ for $i \neq u, i \neq u+1, J_{u}(P)^{u}=J_{u}(P)^{u+1}=P, d_{J_{u}(P)}^{u}=$ $i d_{P}$. The objects $J_{u}(P)$ are projectives and injectives in $C(\operatorname{Proj} \Lambda)$.

For integers $m, n$ with $n \geq m+1$ and $P$ a projective $\Lambda$-module we define the following complexes in $C^{[m, n]}(\operatorname{Proj} \Lambda)$ :

$S(P)$ given by $S(P)^{i}=0$ for $i \neq m, S(P)^{m}=P ; T(P)$ defined by $T(P)^{i}=0$ for $i \neq n, T(P)^{n}=P$. The projectives in $C^{[m, n]}(\operatorname{Proj} \Lambda)$ are the objects $J_{u}(P)$, $u \in[m, n-1]$ and $T(P)$, the injectives in $C^{[m, n]}(\operatorname{Proj} \Lambda)$ are the complexes $J_{u}(P), u \in[m, n-1]$ and $S(P)$ (see corollary 3.3 of $\left.[4]\right)$. For $Y \in C^{[m, n]}(\operatorname{Proj} \Lambda)$ we have the $\mathcal{E}$-sequence:

$$
Y \rightarrow \oplus_{u=n-1}^{m-1} J_{u}\left(Y^{u+1}\right) \rightarrow Y[1],
$$

Taking $l_{m}$ of the above sequence we obtain the $\mathcal{E}$-sequence in $C^{[m, n]}(\operatorname{Proj} \Lambda)$ :

$$
Y \rightarrow S\left(Y^{m}\right) \oplus_{u=m}^{n-1} J_{u}\left(Y^{u+1}\right) \rightarrow l_{m}(Y[1]) .
$$

Observe that if $W$ is an injective in $C^{[m, n]}(\operatorname{Proj} \Lambda)$, any morphism $h$ : $Y \rightarrow W$ is the sum of morphisms factorized through $S\left(Y^{m}\right)$ or through some $J_{u}\left(Y^{u+1}\right)$, for $u \in[m, n-1]$. For $X, Y \in C^{[m, n]}(\operatorname{Proj} \Lambda)$, we denote by $I(X, Y)$ the subspace of morphisms which are factorized through injectives. The space $I(X, Y)$ is generated as $k$-module by the morphisms which are factorized through objects of the form $S(P)$ or $J_{u}(P)$ for $u \in[m, n-1]$.

We denote by $\overline{C^{[m, n]}}(\operatorname{Proj} \Lambda)$ the category with the same objects as those of $C^{[m, n]}(\operatorname{Proj} \Lambda)$ and morphisms the morphisms in $C^{[m, n]}(\operatorname{Proj} \Lambda)$ modulo those which are factorized through injectives. The homomorphisms from $X$ to $Y$ in

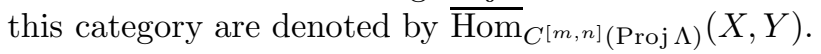


Lemma 4.6 Let $Y \in C^{[m, n]}(\operatorname{Proj} \Lambda)$ with $H^{m+1}(Y)=0$. Take $Y \stackrel{u}{\rightarrow} W \stackrel{v}{\rightarrow} Y[1]$ be an $\mathcal{E}$-sequence in $C^{[m, n]}(\operatorname{Proj} \Lambda)$ with $W$ injective. Then we have:

$$
\operatorname{Ext}_{C^{[m, n]}(\operatorname{Proj} \Lambda)}^{1}(X, Y) \cong \overline{\operatorname{Hom}}_{C^{[m, n]}(\operatorname{Proj} \Lambda)}(X, Y[1]) .
$$

Proof. We have the exact sequence of $k$-modules: $\operatorname{Hom}_{C[m, n](\operatorname{Proj} \Lambda)}(X, W)$ $\stackrel{\operatorname{Hom}(1, v)}{\longrightarrow} \operatorname{Hom}_{C^{[m, n]}(\operatorname{Proj} \Lambda)}(X, Y[1]) \rightarrow \operatorname{Ext}_{C^{[m, n]}(\operatorname{Proj} \Lambda)}^{1}(X, Y) \rightarrow 0$.

For proving our claim we prove that $\operatorname{ImHom}(1, v)=I(X, Y[1])$. For this we only need to prove that a morphism $h: X \rightarrow Y[1]$ which factorizes through $S(P)$ is in the image of $\operatorname{Hom}(1, v)$. But any morphism $h_{1}: S(P) \rightarrow Y[1]$ is factorized by $v$ if $\operatorname{Ext}_{C[m, n](\operatorname{Proj} \Lambda)}^{1}(S(P), Y)=0$. Now $\operatorname{Ext}_{C^{[m, n]}(\operatorname{Proj} \Lambda)}^{1}(S(P), Y) \cong$ $\operatorname{Ext}_{C(\operatorname{Proj} \Lambda)}^{1}(S(P), Y) \cong \operatorname{Hom}_{K}(S(P), Y[1])$. Take $h: S(P) \rightarrow Y[1]$ a morphism of complexes then $h^{m}: P \rightarrow Y^{m+1}$ is such that $d_{Y}^{m+1} h^{m}=0$. Since $H^{m+1}(Y)=0$, there is a $g: P \rightarrow Y^{m}$ with $d_{Y}^{m} g=h^{m}$. This implies that $h$ is homotopic to zero. Consequently $\operatorname{Hom}_{K}(S(P), Y[1])=0$, proving our claim.

Proposition 4.7 Let $W$ and $Z$ be complexes in $C \leq 0(\operatorname{Proj} \Lambda)$ with $H^{i}(W)=0$ and $H^{i}(Z)=0$ for $i \leq-t$ for some positive integer number $t$. Then, for $j>0$ and $m \geq 0$

$$
\operatorname{Ext}_{C(\operatorname{Proj} \Lambda)}^{j}(W, Z) \cong \operatorname{Ext}_{C(\operatorname{Proj} \Lambda)}^{1}\left(l_{-(j+t+m)} W,\left(l_{-(1+t+m)} Z\right)[j-1]\right)
$$

as $\operatorname{End}_{C(\operatorname{Proj} \Lambda)}(Z)-\operatorname{End}_{C(\operatorname{Proj} \Lambda)}(W)$-bimodules.

Proof. We denote by $\mathcal{L}^{[-s, 0]}$ the full subcategory of $K^{\leq 0}(\operatorname{Proj} \Lambda)$, the homotopy category of $C^{\leq 0}(\operatorname{Proj} \Lambda)$, whose objects are those $X$ such that $H^{i}(X)=$ 0 for $i \leq-s$. We recall (see for instance Corollary 5.7 of [4]) that $l_{-s}$ induces an equivalence:

$$
l_{-s}: \mathcal{L}^{[-s, 0]} \rightarrow \overline{C^{[-s, 0]}}(\operatorname{Proj} \Lambda) .
$$

For $s \geq j, l_{-t-s} W, l_{-t-s}(Z[j])$ are in $\mathcal{L}^{[-t-s, 0]}$, then $\operatorname{Ext}_{C(\operatorname{Proj} \Lambda)}^{j}(W, Z) \cong$ $\operatorname{Hom}_{K}(W, Z[j]) \cong \overline{\operatorname{Hom}}_{C[-t-s, 0](\operatorname{Proj} \Lambda)}\left(l_{-t-s} W, l_{-t-s}(Z[j])\right)$. Observe we have $l_{-s-t}(Z[j])=\left(l_{-s-t+j} Z\right)[j]=l_{-t-s}\left[\left(l_{-t-s+j-1} Z\right)[j]\right]$. Thus:

$$
\operatorname{Ext}_{C(\operatorname{Proj} \Lambda)}^{j}(W, Z) \cong \overline{\operatorname{Hom}}_{C-t-s, 0]}(\operatorname{Proj} \Lambda)\left(l_{-t-s} W, l_{-t-s}\left[\left(l_{-t-s+j-1} Z[j]\right]\right)\right.
$$

Now $\left(l_{-t-s+j-1} Z\right)[j]=\left(l_{-t-s+j-1} Z\right)[j-1][1]$. We have that the complex $\left(l_{-t-s+j-1} Z\right)[j-1] \in C^{[-t-s, 0]}(\operatorname{Proj} \Lambda)$. Moreover $\left(\left(l_{-t-s+j-1} Z\right)[j-1]\right)^{-t-s}=$ $Z^{-t-s+j-1}$, and $\left(\left(l_{-t-s+j-1} Z\right)[j-1]\right)^{-t-s+1}=Z^{-t-s+j}$. Since $s \geq j,-t-s+$ $j=-t-(s-j) \leq-t$. Therefore $\left.H^{-t-s+1}\left(\left(l_{-t-s+j-1} Z\right)[j-1]\right)\right)=0$. Then by our previous lemma we have:

$$
\begin{aligned}
& \operatorname{Ext}_{C(\operatorname{Proj} \Lambda)}^{j}(W, Z) \cong \\
& \overline{\operatorname{Hom}}_{C[-t-s, 0]}(\operatorname{Proj} \Lambda) \\
& \cong \operatorname{Ext}_{C-t-s}^{1} W, l_{-t-s}\left[\left(l_{-t-s+j-1} Z[j-1][1]\right]\right) \\
& \cong \operatorname{Ext}_{C(\operatorname{Proj} \Lambda)}^{1}\left(l_{-s-t} W,\left(l_{-t-s+j-1} Z\right)[j-1]\right) .
\end{aligned}
$$


Taking $s=j+m$ we obtain our result.

For $M \in \bmod \Lambda$ we choose a minimal projective resolution:

$$
\ldots \rightarrow P_{M}^{-m} \stackrel{d_{M}^{-m}}{\rightarrow} P_{M}^{-m+1} \stackrel{d_{M}^{-m+1}}{\rightarrow} \ldots P_{M}^{-1} \stackrel{d_{M}^{-1}}{\rightarrow} P_{M}^{0} \stackrel{\eta_{M}}{\rightarrow} M \rightarrow 0
$$

We denote by $P_{M}$ the complex in $C^{\leq 0}(\operatorname{proj} \Lambda)$ :

$$
\ldots \rightarrow P_{M}^{-m} \stackrel{d_{M}^{-m}}{\rightarrow} P_{M}^{-m+1} \stackrel{d_{M}^{-m+1}}{\rightarrow} \ldots P_{M}^{-1} \stackrel{d_{M}^{-1}}{\rightarrow} P_{M}^{0} \rightarrow 0 \rightarrow 0 \ldots
$$

Corollary 4.8 Let $L$ and $N$ be $\Lambda$-modules and $P_{L}, P_{N}$ as above. Then, for $j \geq 0$ and $m \geq 0$

$$
\operatorname{Ext}_{\Lambda}^{j}(M, N) \cong \operatorname{Ext}_{C(\operatorname{Proj} \Lambda)}\left(l_{-(1+j+m)}\left(P_{M}\right), l_{-(2+m)}\left(P_{N}\right)[j-1]\right) .
$$

as $\operatorname{End}_{\Lambda}(M)-\operatorname{End}_{\Lambda}(N)$-bimodules.

Proof. We know that

$$
\operatorname{Ext}_{\Lambda}^{j}(M, N) \cong \operatorname{Hom}_{K}\left(P_{M}, P_{N}[j]\right) \cong \operatorname{Ext}_{C(\operatorname{Proj} \Lambda)}^{j}\left(P_{M}, P_{N}\right) .
$$

Now in proposition 4.7 put $W=P_{M}, Z=P_{N}$, then $t=1$ and we obtain our result.

We will need the following results.

Lemma 4.9 Suppose $Y \in C^{[-m+1,0]}(\Lambda-\operatorname{proj})$ is such that $\operatorname{Im} d_{Y}^{i} \subset \operatorname{rad} Y^{i+1}$ for all $i \in \mathbb{Z}$ and $\operatorname{dim}_{k} H^{j}(Y) \leq c$ for all $j$ and for some $u \in[-m+2, \ldots, 0]$, $\operatorname{dim}_{k} Y^{u} \leq d_{u}$, then $\operatorname{dim}_{k} Y^{u-1} \leq\left(d_{u}+c\right) L$, with $L=\operatorname{dim}_{k} \Lambda$.

Proof. We have $\operatorname{dim}_{k} Y^{u-1} / \operatorname{Ker} d_{Y}^{u-1}=\operatorname{dim}_{k} \operatorname{Im} d_{Y}^{u-1} \leq d_{u}$, moreover we know that $\operatorname{dim}_{k} \operatorname{Ker} d_{Y}^{u-1} / \operatorname{Im} d_{Y}^{u-2} \leq c$. Therefore $\operatorname{dim}_{k} Y^{u-1} / \operatorname{Im} d_{Y}^{u-2} \leq c+d_{u}$.

Here $\operatorname{Im} d_{Y}^{u-2} \subset \operatorname{rad} Y^{u-1}$, thus $\operatorname{dim}_{k} Y^{u-1} / \operatorname{rad} Y^{u-1} \leq \operatorname{dim}_{k} Y^{u-1} / \operatorname{Im} d_{Y}^{u-2}$. Consequently, $\operatorname{dim}_{k} Y^{u-1} \leq\left(c+d_{u}\right) L$.

Lemma 4.10 Let $Y \in C^{[-m+1,0]}(\operatorname{proj} \Lambda)$, with $\operatorname{Im} d_{Y}^{i} \subset \operatorname{rad} Y^{i+1}$ for all $i \in \mathbb{Z}$, such that for all $j \in \mathbb{Z}$, we have the inequality $\operatorname{dim}_{k} H^{j}(Y) \leq c$ for some fixed $c$. Then

$$
\operatorname{dim}_{k} Y \leq c\left(m L+(m-1) L^{2}+(m-2) L^{3}+\ldots+2 L^{m-1}+L^{m}\right) .
$$

Proof. Here $Y^{1}=0$, then by our previous lemma, $\operatorname{dim}_{k} Y^{0} \leq c L$. Then again by lemma 4.9 we have, $\operatorname{dim}_{k} Y^{-1} \leq c\left(L+L^{2}\right), \operatorname{dim}_{k} Y^{-2} \leq c\left(L+L^{2}+L^{3}\right)$, $\ldots, \operatorname{dim}_{k} Y^{-m+1} \leq c\left(L+L^{2}+\ldots+L^{m}\right)$. From here we obtain our result.

Theorem 4.11 (See corollary 9 in [9]) Let $\Lambda$ be a finite-dimensional algebra over an algebraically closed field $k$ and $\mathbf{d}=\left(d_{i}\right)_{i \in \mathbb{Z}}$ be a collection of non-negative 
integers with almost all $d_{i}=0$. Then the family $\mathcal{U}(\mathbf{d})$ of objects $X \in \mathcal{D}^{b}(\Lambda)$ such that $\operatorname{dim}_{k} H^{i}(X)=d_{i}$ for all $i \in \mathbb{Z}$ and $\operatorname{Hom}_{\mathcal{D}^{b}(\Lambda)}(X, X[1])=0$ has only a finite number of isomorphism classes in $\mathcal{D}^{b}(\Lambda)$.

Proof. We may assume $d_{i}=0$ for all $i \leq t$ and $i>0$. Consider now the family $\mathcal{V}(\mathbf{d})$ of those $P \in C^{\leq 0}(\operatorname{proj} \Lambda)$ such that $\operatorname{dim}_{k} H^{i}(P)=d_{i}$ for all $i \in \mathbb{Z}$, $\operatorname{Hom}_{K}(P, P[1])=0$ and for all $i \in \mathbb{Z}, \operatorname{Im} d_{P}^{i} \subset \operatorname{rad} P^{i+1}$. For each $X \in \mathcal{U}(\mathbf{d})$ we may choose a quasi-isomorphism $P_{X} \rightarrow X$ with $P_{X} \in C^{\leq 0}(\operatorname{proj} \Lambda)$.

We have $\operatorname{Hom}_{K}\left(P_{X}, P_{X}[1]\right) \cong \operatorname{Hom}_{\mathcal{D}^{b}(\Lambda)}(X, X[1])$. Clearly the assignament $X \mapsto P_{X}$ stablishes a bijection between the isomorphism classes of $\mathcal{U}(\mathbf{d})$ and those of $\mathcal{V}(\mathbf{d})$. If $P \in \mathcal{V}(\mathbf{d})$, then $P \in \mathcal{L}^{[-t-1,0]}$.

By proposition 4.7 for $P \in \mathcal{V}(\mathbf{d})$ we have: $0=\operatorname{Hom}_{K}(P, P[1]) \cong$ $\operatorname{Ext}_{C(\operatorname{Proj} \Lambda)}^{1}(P, P) \cong \operatorname{Ext}_{C{ }^{[-t-1,0]}(\operatorname{Proj} \Lambda)}^{1}\left(l_{-t-1} P, l_{-t-1} P\right)$.

Using Lemma 4.10 one can prove that there is a number $n(\mathbf{d})$, such that if $P \in \mathcal{V}(\mathbf{d})$ then $\sum_{i \in \mathbb{Z}} \operatorname{dim}_{k}\left(l_{-t-1} P\right)^{i} \leq n(\mathbf{d})$. Therefore the functor $l_{-t-1}$ induces a bijection between the isomorphism classes of $\mathcal{V}(\mathbf{d})$ and the isomorphism classes of a subfamily of the family $\mathcal{F}(n(\mathbf{d}))$ consisting of the complexes $Z \in C^{[-t-1,0]}(\operatorname{proj} \Lambda)$ which have not injectives in this category as direct summands, $\operatorname{Ext}_{C{ }^{[-t-1,0]}(\operatorname{proj} \Lambda)}^{1}(Z, Z)=0$, and $\sum_{i \in \mathbb{Z}} \operatorname{dim}_{k}(Z)^{i} \leq n(\mathbf{d})$.

The category $C^{[-t-1,0]}(\operatorname{Mod} \Lambda)$ is an abelian category with enough projectives, the projectives in this category are the complexes $T(P), J_{u}(P), u \in$ $[-t-1,-1]$ introduced before. Then taking $H=\oplus_{u=-t-1}^{-1} J_{u}(\Lambda) \oplus T(\Lambda)$ and $\Gamma=\operatorname{End}_{C[-t-1,0]}(\operatorname{Mod} \Lambda)(H)$, the functor

$$
F=\operatorname{Hom}_{C}^{[-t-1,0]}(\operatorname{Mod} \Lambda)(H,-): C^{[-t-1,0]}(\operatorname{Mod} \Lambda) \rightarrow \operatorname{Mod} \Gamma
$$

is an equivalence of abelian categories.

Now there is a number $m(\mathbf{d})$ such that for all $Z \in \mathcal{F}(n(\mathbf{d})), \operatorname{dim}_{k} F(Z) \leq$ $m(\mathbf{d})$.

Since the category $C^{[-t-1,0]}(\operatorname{proj} \Lambda)$ is a full subcategory of the category $C^{[-t-1,0]}(\operatorname{Mod} \Lambda)$, closed under extensions, then for $Z \in \mathcal{F}(n(\mathbf{d}))$,

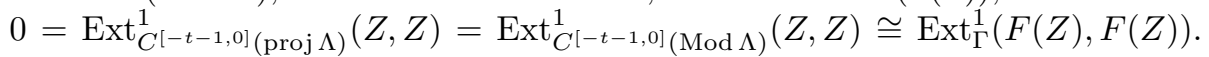
Therefore $F$ gives a bijection between the isomorphism classes of $\mathcal{F}(n(\mathbf{d}))$ and the isomorphism classes of a family of $\Gamma$-modules $M$ with $\operatorname{dim}_{k} M \leq m(\mathbf{d})$ and $\operatorname{Ext}_{\Gamma}(M, M)=0$. But by a result of D. Voigt ([10]), this last family has only a finite number of isomorphism classes. This implies that our family $\mathcal{U}(\mathbf{d})$ has only a finite number of isomorphism classes.

\section{An application to modules}

Let $\Lambda$ be an artin algebra over a commutative artinian ring $k$. In this section we study under which conditions two finitely generated $\Lambda$-modules $M$ and $N$ with $\operatorname{Ext}_{\Lambda}(M, M)=0$ and $\operatorname{Ext}_{\Lambda}(N, N)=0$ are isomorphic. As before for $M \in \bmod \Lambda$ 
we choose a minimal projective resolution:

$$
\ldots \rightarrow P_{M}^{-m} \stackrel{d_{M}^{-m}}{\rightarrow} P_{M}^{-m+1} \stackrel{d_{M}^{-m+1}}{\rightarrow} \ldots P_{M}^{-1} \stackrel{d_{M}^{-1}}{\rightarrow} P_{M}^{0} \stackrel{\eta_{M}}{\rightarrow} M \rightarrow 0 .
$$

We denote by $P_{M}$ the complex in $C^{\leq 0}(\operatorname{proj} \Lambda)$ :

$$
\ldots \rightarrow P_{M}^{-m} \stackrel{d_{M}^{-m}}{\rightarrow} P_{M}^{-m+1} \stackrel{d_{M}^{-m+1}}{\rightarrow} \quad \ldots P_{M}^{-1} \stackrel{d_{M}^{-1}}{\rightarrow} P_{M}^{0} \rightarrow 0 \rightarrow 0 \ldots
$$

For $M \in \bmod \Lambda$ we put $\Omega(M)=\operatorname{Ker}\left(\eta_{M}\right)$.

Theorem 5.1 Let $M$ and $N$ be in $\bmod \Lambda$ such that $\operatorname{Ext}_{\Lambda}^{1}(M, M)=0$ and $\operatorname{Ext}_{\Lambda}^{1}(N, N)=0$. Then $M \cong N$ if and only if $M / \operatorname{rad} M \cong N / \operatorname{rad} N$ and $\Omega(M) \cong$ $\Omega(N)$.

Proof. Neccesity is obvious, let us to prove sufficiency.

Let $P_{M}^{\prime}=l_{-2}\left(P_{M}\right)$ and $P_{N}^{\prime}=l_{-2}\left(P_{N}\right)$.

By lemma 4.5 we have an exact isomorphism of categories

$$
\urcorner_{-1}: C(\operatorname{Proj} \Lambda) \rightarrow M \operatorname{orph}\left(C^{\leq-1}(\operatorname{Proj} \Lambda), C^{\geq-1}(\operatorname{Proj} \Lambda)\right)
$$

wich induces an exact isomorphism of categories

$$
\urcorner: C^{[-2,0]}(\operatorname{Proj} \Lambda) \rightarrow \operatorname{Morph}\left(C^{[-2,-1]}(\operatorname{Proj} \Lambda), C^{[-1,-1]}(\operatorname{Proj} \Lambda)\right)
$$

We denote by $\mathcal{M}_{2}$ the category $\operatorname{Morph}\left(C^{[-2,-1]}(\operatorname{Proj} \Lambda), C^{[-1,-1]}(\operatorname{Proj} \Lambda)\right)$. By corollary 4.8 we have $\operatorname{Ext}_{C(\operatorname{Proj} \Lambda)}^{1}\left(P_{M}^{\prime}, P_{M}^{\prime}\right) \cong \operatorname{Ext}_{\Lambda}^{1}(M, M)=0$. Then

$$
\text { (1) } \left.\operatorname{Ext}_{\mathcal{M}_{2}}\left(\neg\left(P_{M}^{\prime}\right),\right\urcorner\left(P_{M}^{\prime}\right)\right)=0 \text {. }
$$

In a similar way

$$
\text { (2) } \left.\operatorname{Ext}_{\mathcal{M}_{2}}\left(\neg\left(P_{N}^{\prime}\right),\right\urcorner\left(P_{N}^{\prime}\right)\right)=0 \text {. }
$$

The categories of bounded complexes over the finitely generated projective $\Lambda$ modules are Krull-Schmidt categories, so using the functors "erase at right" and "pull and erase" we get $t_{-1}\left(P_{M}^{\prime}\right) \cong \oplus_{i=1}^{u} a_{i} X_{i}$ and $s_{-1}\left(P_{M}^{\prime}\right) \cong \oplus_{j=1}^{v} b_{j} Y_{j}$, $t_{-1}\left(P_{N}^{\prime}\right) \cong \oplus_{i=1}^{u} c_{i} X_{i} s_{-1}\left(P_{N}^{\prime}\right) \cong \oplus_{j=1}^{v} h_{j} Y_{j}$, where all decompositions are sums of pairwise non-isomorphic indecomposable objects. Now $t_{-1}\left(P_{M}^{\prime}\right)$ and $t_{-1}\left(P_{N}^{\prime}\right)$ correspond to minimal projective resolutions of $\Omega(M)$ and $\Omega(N)$ respectively, since $\Omega(M) \cong \Omega(N)$ then $a_{i}=c_{i}$ for all $i \in\{1, \ldots, v\}$. On the other hand the only non zero module in the complexes $s_{-1}\left(P_{M}^{\prime}\right)$ and $s_{-1}\left(P_{M}^{\prime}\right)$ are $P_{M}^{0}$ and $P_{N}^{0}$ respectively, since $M / \operatorname{rad} M \cong N / \operatorname{rad} N, P_{M}^{0} \cong P_{N}^{0}$. Therefore $s_{-1}\left(P_{M}^{\prime}\right) \cong$ $s_{-1}\left(P_{N}^{\prime}\right)$ and this implies that $b_{j}=h_{j}$ for $j \in\{1, \ldots, u\}$. Let $X=\oplus_{i=1}^{u} X_{i}$ and $Y=\oplus_{j=1}^{v} Y_{j}$.

By proposition 3.7 we have an exact equivalence of categories

$$
\Theta: M \operatorname{orph}(\operatorname{addX}, \operatorname{add} Y) \rightarrow \xi_{Y}^{X}\left(R_{Y}^{X}\right)
$$

Then by (1) and (2) we have: 
$\left.\left.\left.\left.\operatorname{Ext}_{\xi_{Y}^{X}\left(R_{Y}^{X}\right)}(\Theta\urcorner\left(P_{M}^{\prime}\right), \Theta\right\urcorner\left(P_{M}^{\prime}\right)\right)=0, \quad \operatorname{Ext}_{\xi_{Y}^{X}\left(R_{Y}^{X}\right)}(\Theta\urcorner\left(P_{N}^{\prime}\right), \Theta\right\urcorner\left(P_{N}^{\prime}\right)\right)=0$.

It follows by 5.1 of [5] that $\left.\Theta\urcorner\left(P_{M}^{\prime}\right) \cong \Theta\right\urcorner\left(P_{N}^{\prime}\right)$. Then $P_{M}^{\prime} \cong P_{N}^{\prime}$ and consequently $M \cong N$.

Theorem 5.2 Assume $M, N$ in $\bmod \Lambda$ have finite projective dimension and $\operatorname{Ext}_{\Lambda}^{1}(M, M) \cong \operatorname{Ext}_{\Lambda}^{1}(N, N)=0$ and, for all $j>1 \operatorname{Ext}_{\Lambda}^{j}\left(M, \Omega^{j-1}(M)\right) \cong$ $\operatorname{Ext}_{\Lambda}^{j}\left(N, \Omega^{j-1}(N)\right)=0$, then $M \cong N$ if and only for all $j, P_{M}^{-j} \cong P_{N}^{-j}$.

Proof. Neccesity is obvious, let us to prove sufficiency by induction on $m=\max (p(M), p(N))$, where $p(M)$ and $p(N)$ are the projective dimension of $M$ and $N$ respectively. If $m=0$ our claim is trivial. Suppose our claim proved for $m-1$, we will prove it for $m$. $\operatorname{But}_{\operatorname{Ext}_{\Lambda}^{1}(\Omega(M), \Omega(M)) \cong \operatorname{Ext}_{\Lambda}^{2}(M, \Omega(M))=0}$ and for all $j>1$, $\operatorname{Ext}_{\Lambda}^{j}\left(\Omega(M), \Omega^{j-1}(\Omega(M))\right) \cong \operatorname{Ext}_{\Lambda}^{j+1}\left(M, \Omega^{j}(M)\right)=0$. Thus $\Omega(M)$ satisfies the hypothesis of our theorem, similarly $\Omega(N)$ also satisfies the hypothesis of our theorem, since $p(\Omega(M))=p(\Omega(N))=m-1$ and $P_{M}^{1} / \operatorname{rad} P_{M}^{1} \cong$ $\Omega(M) / \operatorname{rad} \Omega(M) \cong P_{N}^{1} / \operatorname{rad} P_{N}^{1} \cong \Omega(N) / \operatorname{rad} \Omega(N)$, by the induction hypothesis, $\Omega(M) \cong \Omega(N)$. Here $P_{M}^{0} \cong P_{N}^{0}$, then by theorem 5.1, we obtain $M \cong N$.

Example 5.3 We present an example of two non-isomorphic modules with both having finite minimal projective resolution and the same projectives. Let $\Lambda$ be the $k$-algebra of the quiver

$$
\circ_{1} \underset{\alpha_{2}}{\stackrel{\alpha_{1}}{\rightrightarrows}} \circ_{2} \underset{\beta_{2}}{\stackrel{\beta_{1}}{\rightrightarrows}} \circ_{3}
$$

with the relation $\left\{\beta_{1} \alpha_{1}, \beta_{2} \alpha_{2}\right\}$. The representations

$$
k \underset{0}{\stackrel{1}{\rightrightarrows}} k \underset{1}{\stackrel{0}{\rightrightarrows}} \quad k \quad k \underset{1}{\stackrel{0}{\rightrightarrows}} \quad k \underset{0}{\stackrel{1}{\rightrightarrows}} \quad k
$$

have trivial selfextensions group but both have a minimal projective resolution with data $\left(\ldots, 0, \ldots, 0, \Lambda e_{3}, \Lambda e_{2}, \Lambda e_{1}\right)$.

Acknowledgements Both authors thank the support of project " $43374 \mathrm{~F}$ " of Fondo Sectorial SEP-Conacyt.

\section{References}

[1] Auslander, M., Reiten, I., Smalø, S. Representation theory of artin algebras (Cambridge studies in advanced mathematics: 36), Cambridge University Press, Cambridge, 1995. 
[2] Bautista, R. The category of morphisms between projectives, Communications in Algebra (11) vol. 32, (2004), 4303-4332.

[3] Bautista, R., Salmerón, L. On discrete and inductive algebras, Preprint (2002). To appear in Fields Institute Communications Series.

[4] Bautista, R., Souto, M.J., Zuazua, R. Almost split sequences for complexes of fixed size, Journal of Algebra (1) vol. 287 (2005), 140-168.

[5] Bautista, R., Zuazua, R. Exact structures for lift categories, Preprint (2002). To appear in Fields Institute Communications Series.

[6] Crawley-Boevey, W.W. Matrix reductions for artinian rings and an application to rings of finite representation type, Journal of Algebra 157, (1993), $1-25$.

[7] Dräxler, P., Reiten, I., Smalø, S., Solberg, O. Exact categories and vector space categories, Trans. Amer. Math. Soc. 351,(1999), 647-682.

[8] Gabriel, P., Roiter, A.V. Representations of finite dimensional algebras. Encyclopaedia of the Mathematical Sciences. 73, Kostrikin, A.I., Shafarevich, I.V. (Eds.), Algebra VIII, Springer (1992).

[9] Huisgen-Zimmermann, B., Saorín, M. Geometry of chain complexes and outer automorphisms under derived equivalence, Trans. Amer. Math. Soc. 353 (2001), 4757-4777.

[10] Voigt, D. Induzierte Darstellungen in der Theorie der endlichen, algebraischen Gruppen, Lecture Notes in Math. 592, Springer-Verlag, Berlin, 1977. MR 58:5949

\section{R. Bautista}

Instituto de Matemticas, UNAM, Campus Morelia

Xangari

raymundo@matmor.unam.mx

E. Pérez

efren_math@yahoo.com.mx

efren_mathe@yahoo.com 